\title{
Association between subtalar articular surface typing and flat foot deformity: which type is more likely to cause flat foot deformity
}

Lei Zhang ${ }^{1,2,3,4 \dagger}$, Xiaoyao Peng ${ }^{5 \dagger}$, Siyuan He ${ }^{6 \dagger}$, Xin Zhou ${ }^{1,2,3,4}$, Gang Yi $i^{1,2,3,4}$, Xiaogao Tang ${ }^{1,2,3,4}$, Bingkun Li ${ }^{1,2,3,4}$, Guoyou Wang ${ }^{1,2,3,4^{*}}$, Wanxue Zhao ${ }^{5}$ and Yuening Yang ${ }^{5}$

\begin{abstract}
Background: Previous studies have shown a wide range of anatomical classifications of the subtalar joint (STJ) in the population and this is related to the different force line structures of the foot. Different subtalar articular surface morphology may affect the occurrence and development of flat foot deformity, and there are fewer studies in this area. The main objective of our study was to determine the association of different subtalar articular surface with the occurrence and severity of flat foot deformity.
\end{abstract}

Methods: We analyzed the imaging data of 289 cases of STJ. The articular surface area, Gissane's angle and Bohler's angle of subtalar articular surface of different types were counted. The occurrence and severity of flat foot deformity in different subtalar articular surface were judged by measuring the Meary angle of foot.

Results: We classified 289 cases of subtalar articular surface into five types according to the morphology. According to Meary angle, the flat foot deformity of Type I and Type IV are significantly severer than Type II $(P<0.05)$. Type II $\left(7.65 \pm 1.38 \mathrm{~cm}^{2}\right)$ was significantly smaller than Type I $\left(8.40 \pm 1.79 \mathrm{~cm}^{2}\right)$ in the total joint facet area $(P<0.05)$. Type III $\left(9.15 \pm 1.92 \mathrm{~cm}^{2}\right)$ was smaller than Type I $\left(8.40 \pm 1.79 \mathrm{~cm}^{2}\right), \|\left(7.65 \pm 1.38 \mathrm{~cm}^{2}\right)$ and IV $\left(7.81 \pm 1.74 \mathrm{~cm}^{2}\right)(P<0.05)$. Type II $\left(28.81 \pm 7.44^{\circ}\right)$ was significantly smaller than Type I (30.80 \pm 4.61 degrees), and IV ( $32.25 \pm 5.02$ degrees) in the Bohler's angle $(P<0.05)$. Type II (128.49 \pm 6.74 degrees) was smaller than Type I (131.58 \pm 7.32 degrees), and IV (131.94 \pm 5.80 degrees) in the Gissane's angle $(P<0.05)$.

Conclusions: After being compared and analyzed the measurement of morphological parameters, joint facet area and fusion of subtalar articular surface were closely related to the severity of flat foot deformity and Type I and IV were more likely to develop severer flat foot deformity.

Level of evidence: Level III, retrospective comparative study.

Keywords: Flat foot, Meary angle, Subtalar joint

*Correspondence: wang_guoyou1981@163.com

${ }^{\dagger}$ Lei Zhang, Xiaoyao Peng, Siyuan He are the co-first authors, they contributed equally to this work.

${ }^{4}$ Clinical Base of The Affiliated Traditional Chinese Medicine Hospital of Southwest Medical University, Guangdong Province Medical 3D Printing Application Transformation Engineering Technology Research Center, Luzhou 646000, China

Full list of author information is available at the end of the article

\section{Background}

Flat foot is a deformity of foot caused by various reasons in clinic which is a disease characterized by abduction of forefoot, valgus of the heel and collapse of medial longitudinal arch of foot [1]. At present, the main known pathogenic factors are posterior tibial tendon dysfunction (PTTD), and the occurrence of external trauma such as calcaneus or talus fracture leading to hind foot original author(s) and the source, provide a link to the Creative Commons licence, and indicate if changes were made. The images or other third party material in this article are included in the article's Creative Commons licence, unless indicated otherwise in a credit line to the material. If material is not included in the article's Creative Commons licence and your intended use is not permitted by statutory regulation or exceeds the permitted use, you will need to obtain permission directly from the copyright holder. To view a copy of this licence, visit http://creativecommons.org/licenses/by/4.0/. The Creative Commons Public Domain Dedication waiver (http://creativeco mmons.org/publicdomain/zero/1.0/) applies to the data made available in this article, unless otherwise stated in a credit line to the data. 
structural disorder, so that changes in medial longitudinal arch structure and force line can also lead to the occurrence of flat foot [2]. Because the physiological structure and biomechanical composition of the foot are relatively complex, the detection and treatment methods of flat foot need further research in clinic [3, 4]. When the structure of the subtalar joint (STJ) between talus and calcaneus is abnormal, the foot force lines will change and affect the occurrence of ankle joint osteoarthritis [5]. As the most dominant load-bearing joint of the hind foot, the STJ combined with talocalcaneal joint bears weight and transmits to the whole foot, which is very important to maintain the stability of the foot structure $[6,7]$. The existing anatomical studies show that the foot arch is mainly composed of transverse arch, lateral longitudinal arch and medial longitudinal arch of foot [8]. The anterior and middle subtalar articular surface of STJ belong to the medial longitudinal arch, and the posterior subtalar articular surface belongs to the lateral longitudinal arch. Therefore, the occurrence of flat foot also involves the change of the anatomical structure of subtalar articular surface. Our research focuses on the relationship between the occurrence and development of flat foot deformity and subtalar articular surface classification.

The study of Kothari et al. proved that the absence of the anterior subtalar articular surface can cause flat foot deformity [9]. When there is a flat foot deformity, the foot is pronated with forefoot abduction, the subtalar joint axis is valgus, and calcaneal valgus results in a change in the force area of the subtalar articular surface. The medially inclined posterior calcaneum articular facets makes the contact force point more concentrated to the lateral part, which may lead to the appearance of lateral subluxation of the STJ [10, 11]. Studies have demonstrated that the assessment of subluxation and discordance in the STJ is an accurate diagnostic tool for acquired flat foot deformity in adults, which may be related to the simple anatomy and small area of the middle subtalar articular surface [12]. And in acquired flat foot deformity, subluxation of the middle articular may better characterize the degree of subluxation of the STJ than that of the posterior STJ [13].

In our previous work, we found that there are extensive anatomical variations of subtalar articular surface in population, which will affect the stress structure of foot and affect the occurrence of osteoarthritis [14]. When the normal anatomical relationship of STJ can be restored, the force line structure of foot can be directly changed and the recovery of midfoot structure can be promoted through the surrounding interosseous ligament, thereby remodels the transverse and longitudinal arches of the foot and relieves a range of symptoms from flat foot [15]. And studies have demonstrated that there is a risk of damage to the subtalar articular surface when performing lateral calcaneal lengthening osteotomy for flat foot without considering the anatomical variation of the STJ [16].

The purpose of this study is to explore the relationship between the different types of subtalar articular surface and the occurrence and severity of flat foot by analyzing the imaging data of STJ, so as to provide the basis for clinical treatment of patients with different types of subtalar articular surface.

\section{Materials and methods Subjects}

All procedures were approved by the Ethical Committee of Affiliated Traditional Chinese Medicine Hospital of Southwest Medical University (No. KY2018043). A total of 207 people with 289 STJs and feet met the inclusion criteria (131 male, 76 female), including foot with varying severity of flat foot deformity. There were 159 left calcaneus and foot cases and 130 right calcaneus and foot cases. The average age of patients is 44 (range, 18-80) years. All patients gave informed consent for the experimentation.

Inclusion criteria: (1) Complete development of calcaneus, (2) No history of degenerative disease, fracture, and surgery involving the ankle, especially the subtalar joint, (3) No major systemic disease, tolerable to computed tomography (CT), X-ray examination, (4) The imaging data and other basic information of patients are complete. All patients were screened by medical practitioners and met the above inclusion criteria.

\section{Methods for classification of subtalar articular surface}

The facets morphology of calcaneus were captured by a spiral CT scanner (Siemens AG, Munich, Free State of Bavaria). The images of STJ were reconstructed in three-dimensional (3D) after CT scanning. The STJs were classified according to the number, absence and shape of calcaneal joint surface which was observed by observers. The subtalar articular surface classification was conducted by two medical practitioners. In case of any divergence between the two medical practitioners regarding the results of the classification, a judgement was made by a third medical practitioner. All medical practitioners had more than 5 years of work experience.

Based on previous studies [17], we classified the subtalar articular surface into the following five types:

Type I: the anterior and middle facets of calcaneus fused in a continuous and spindle shape facet, and separated from the posterior facet.

Type II: the anterior, middle, and posterior facets of calcaneus were present independently. 
Type III: the anterior facet of calcaneus was absent, and the middle and posterior facets were present independently.

Type IV: the anterior and middle facets of calcaneus fused in a continuous and calabash shape facet, and separated from the posterior facets.

Type V: the anterior facet of calcaneus was absent, and the middle and posterior facets were fused in a continuous facet (Fig. 1).

\section{The morphological parameters of measurement}

As each joint facet is approximated as a rectangle, the joint facet area is calculated by using the short and long axes of each facet. The total joint facet area is obtained by adding up each small facet area (Fig. 2).

The data of angle measurement were from lateral weightbearing radiographs (Siemens AG, Munich, Free State of Bavaria):

Gissane's angle: The angle is formed by the anterior facet and the posterior facet. When the anterior process joint is missing, it was measured by replacing it with the upper surface of the anterior process of calcaneus.

Bohler's angle: The angle is measured by the line from the highest point of the posterior facet to the highest point of the anterior process of calcaneus and the line from the upper edge of the calcaneal tubercle to the tangent point of the posterior facet.

Meary angle: The angle is formed by the long axis of the first metatarsal bone (the line between the distal midpoint and proximal midpoint of the first metatarsal diaphysis) and the central axis of talus (the line between the midpoint of the upper and lower surface of the middle talus and the midpoint of the talus neck). When the measurement is complete, the flat foot deformity is graded according to the following criteria (degree) [18]:

Mild: [4,15).

Moderate: $[15,30)$.

Severe: $[30,+\infty)$.

\section{Statistical methods}

Categorical variables of the articular facets of the calcaneus were recorded as numbers. All data were presented as the mean and standard deviation (SD). Morphological parameters of measurement of five types of the subtalar articular surface were compared by One-way ANOVA and the statistically significance was considered at $P \leq 0.05$. Rank sum test was used to compare the severity of flat foot deformity and the statistically significance

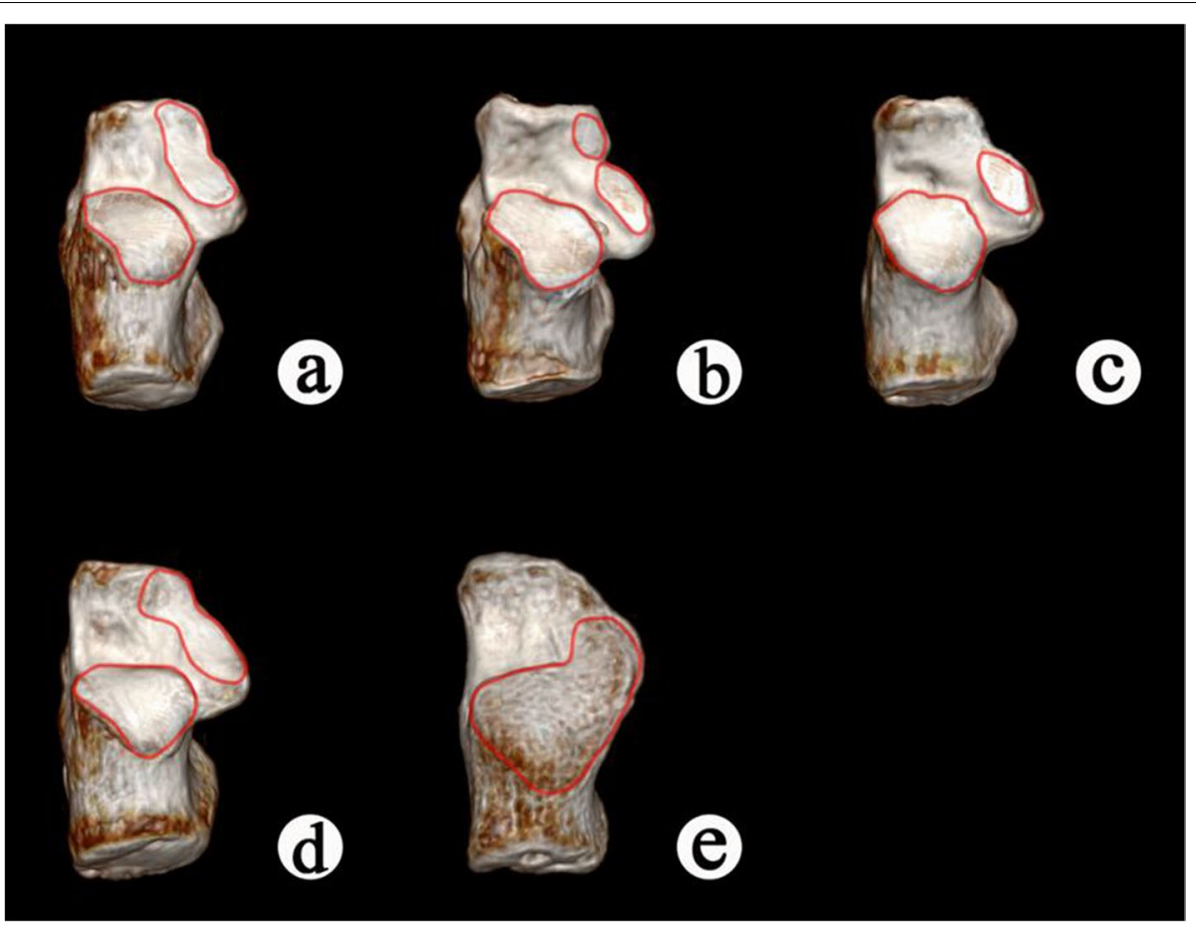

Fig. 1 3D reconstruction of subtalar articular surface after CT scanning. a Type I, the anterior and middle facets of calcaneus fused in a continuous and spindle shape facet, and separated from the posterior facet. $\mathbf{b}$ Type II, the anterior, middle, and posterior facets of calcaneus were present independently. c Type III, the anterior facet of calcaneus was absent, and the middle and posterior facets were present independently. $\mathbf{d}$ Type IV, the anterior and middle facets of calcaneus fused in a continuous and calabash shape facet, and separated from the posterior facets. e Type $V$, the anterior facet of calcaneus was absent, and the middle and posterior facets were fused in a continuous facet 


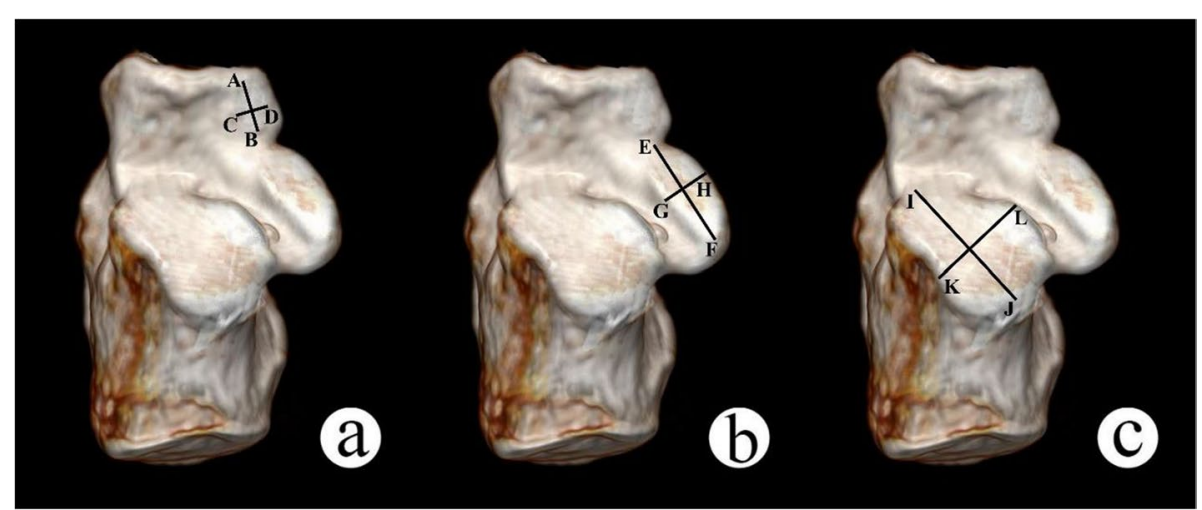

Fig. 2 The measurement of total joint facet area. a AB: the long-axis of the anterior facet of calcaneus, CD: the short-axis of the anterior facet of calcaneus; $\mathbf{b}$ EF: the long-axis of the middle facet of calcaneus, GH: the short-axis of the middle facet of calcaneus; $\mathbf{c}$ IJ: the long-axis of the posterior facet of calcaneus, $\mathrm{KL}$ : the short-axis of the posterior facet of calcaneus. Total joint facet area $=A B^{*} \mathrm{CD}+\mathrm{EF}{ }^{*} \mathrm{GH}+\mathrm{IJ} \mathrm{J}^{*} \mathrm{KL}$

was considered at $P \leq 0.05$. The intraclass correlation coefficient (ICC) and 95\% confidence interval (95\% CI) were used to express the test-retest reliability, and the statistically significance was considered at $P \leq 0.05$. SPSS version 25.0(IBM Corp, Armonk, NY) for Windows software was used for all statistical analysis.

\section{Result}

We classified the subtalar articular surface into five types based on different characteristics of the calcaneal articular surface morphology, and noted that there were distinct differences in each type: Type I, the anterior and middle facets of calcaneus fused in a continuous and spindle shape facet, and separated from the posterior calcaneus facet; Type II, the anterior, middle, and posterior facets of calcaneus were present independently; Type III, the anterior facet of calcaneus was absent, and the middle and posterior facets were present independently; Type $\mathrm{IV}$, the anterior and middle facets of calcaneus fused in a continuous and calabash shape facet, and separated from the posterior facets; Type V, the anterior calcaneus facet was absent, and the middle and posterior facets were fused in a continuous facet (Fig. 1).

We graded the severity of flat foot by the angle of Meary angle (degree): Mild flat-foot, [4,15); Moderate flat-foot, [15,30); Severe flat-foot, $[30,+\infty)$. According to Table 1, of the 143 Type I subtalar articular surfaces, 14 had normal feet, 73 had mild flat foot deformity, 54 had moderate flat foot deformity and 2 had severe flat foot deformity. Of the 31 Type IV subtalar articular surfaces, 3 had normal feet, 19 had mild flat foot deformity, and 9 had moderate flat foot deformity. Of the 61 Type II subtalar articular surfaces, 17 had normal feet, 33 had mild flat foot deformity, and 11 had moderate flat foot deformity, and it had significantly less severity of flat foot deformity
Table 1 Comparison of the severity of flat foot deformity in different types of subtalar articular surface

\begin{tabular}{llllll}
\hline & No. & Normal & Mild & Moderate & Severe \\
\hline Type I & 143 & 14 & 73 & 54 & 2 \\
Type II $^{*}$ & 61 & 17 & 33 & 11 & 0 \\
Type III $^{*}$ & 47 & 12 & 23 & 12 & 0 \\
Type IV $^{* *}$ & 31 & 3 & 19 & 9 & 0 \\
Type V & 7 & 1 & 4 & 2 & 0 \\
\hline
\end{tabular}

Note: ${ }^{*} p<0.05$ vs Type I, ${ }^{* *} p<0.05$ vs Type II. ICC $=0.918[95 \% \mathrm{Cl}=0.898-0.935]$ $(P<0.001)$

than Type I and Type IV $(P<0.05)$ (Table 1$)$. The testretest reliability of two medical practitioners was excellent: $\mathrm{ICC}=0.918[95 \% \mathrm{CI}=0.898-0.935](P<0.001)$.

In terms of total joint facet area, Type II $\left(7.65 \pm 1.38 \mathrm{~cm}^{2}\right) \quad$ was significantly smaller than Type I $\left(8.40 \pm 1.79 \mathrm{~cm}^{2}\right) \quad(P<0.05)$. Besides, Type III $\left(9.15 \pm 1.92 \mathrm{~cm}^{2}\right)$ was significantly smaller than Type I $\left(8.40 \pm 1.79 \mathrm{~cm}^{2}\right)$, II $\left(7.65 \pm 1.38 \mathrm{~cm}^{2}\right)$ and IV $\left(7.81 \pm 1.74 \mathrm{~cm}^{2}\right)(P<0.05)$ (Table 2$)$.

Table 2 Calcaneus morphological parameters based on classification

\begin{tabular}{llll}
\hline & $\begin{array}{l}\text { Average total joint } \\
\text { facet area }\left(\mathbf{c m}^{2}\right)\end{array}$ & $\begin{array}{l}\text { Mean Gissane's } \\
\text { angle (degree) }\end{array}$ & $\begin{array}{l}\text { Mean Bohler's } \\
\text { angle (degree) }\end{array}$ \\
\hline Type I & $8.40 \pm 1.79$ & $131.58 \pm 7.32$ & $30.80 \pm 4.61$ \\
Type II & $7.65 \pm 1.38^{*}$ & $128.49 \pm 6.74^{*}$ & $28.81 \pm 7.44^{*}$ \\
Type III & $6.93 \pm 1.08^{* * * *}$ & $130.73 \pm 6.36$ & $30.80 \pm 4.69$ \\
Type IV & $7.81 \pm 1.74^{* * *}$ & $131.94 \pm 5.80^{* *}$ & $32.25 \pm 5.02^{* *}$ \\
Type V & $8.18 \pm 2.83$ & $130.80 \pm 3.73$ & $31.90 \pm 7.16$ \\
\hline
\end{tabular}

Note: Data were presented as the mean and SD. ${ }^{*} p<0.05$ vs Type ${ }^{* *} p<0.05$ vs Type II, ${ }^{* * *} p<0.05$ vs Type III 
Type II (28.81 \pm 7.44 degrees) was significantly smaller than Type I ( $30.80 \pm 4.61$ degrees), and IV ( $32.25 \pm 5.02$ degrees) in the Bohler's angle $(P<0.05)$ (Table 2$)$.

Type II (128.49 \pm 6.74 degrees) was statistically smaller than Type I (131.58 \pm 7.32 degrees), and IV $(131.94 \pm 5.80$ degrees) in the Gissane's angle $(P<0.05)$ (Table 2$)$.

\section{Discussion}

The etiology and features of flat foot were considered in the original studies to be PTTD, but as research progressed, the widely accepted view was that flat foot was a progressive deformity associated with a variety of structures $[19,20]$. The clinical symptoms that manifest as the deformity progresses can also vary and can result from an initial pain in the medial arch affecting a normal gait ultimately progressing to severe conditions such as arthritis due to abnormalities in the force placed on the articular surface [21-24]. Found that the bony support to the talus differed among the different types of the subtalar articular surface, and that stabilization of the STJ when support decreased or when mobility increased would be more dependent on the surrounding ligamentous structures, with extension of time after the ligamentous stretch resulting in decreased static stability of the joint and increased potential pathological activity, giving rise to manifestations of flat foot deformities such as decreased medial longitudinal arch $[9,25,26]$.

In our study, we utilized the CT 3D reconstruction technique to measure and type morphological parameters of the subtalar articular surface. In view of the high resolution and 3D reconstruction of Standing (Weightbearing) CT views, it can provide more and accurate measurement parameters for borderline Meary categories compared with Standing (Weightbearing) X-rays, but it still cannot completely replace the X-rays in the process of clinical diagnosis and treatment. All $289 \mathrm{cal}-$ caneus were classified into five types according to the articular surface morphological characteristics, and our results showed that the most common was Type I, followed by Type II, III, IV, and V in that order. We indexed the severity of flat foot according to Meary angle in five types of subtalar articular surfaces and found that Type I and IV had significantly higher severity than Type II $(P<0.05)$ (Table 1$)$. Approximately $15 \%$ will develop flat foot due to developmental problems, but $7-15 \%$ will develop clinical symptoms, meanwhile, the irregular shape of the subtalar articular surface can affect its stability and movement, so the severity of flat foot may be related to the type of subtalar articular surface, while our study showed that Type I and IV were associated with severer flat foot deformity $[27,28]$.

The key factors affecting the mobility of the STJ are the area of the articular surface and fusion, and a larger and flatter articular surface is associated with greater joint mobility $[17,29]$. The total area of the subtalar articular surface was significantly larger in Type I than in Type II $(P<0.05)$ (Table 2$)$. We think that this is because the anterior and middle articular surfaces of the Type I subtalar articular surface were fusing in a spindle shape, while all three articular surfaces of the Type II articular surface existed independently, and this result makes the talus of Type I more mobile relative to the calcaneus and more likely to cause the occurrence of flat foot deformity by causing the alteration of the surrounding ligamentous structures. The triangle formed from three separate articular surfaces according to previous descriptions of Type II constitutes an "osseous tripod" and is therefore best stabilized in all types $[26,30]$. The flat foot deformity of Type IV is severer compared with Type II, but there is no obvious difference in the area of the articular surface between these two types, and the reason for this may be because the anterior articular surface and middle articular surface of Type IV are fused in a calabash shape and those of Type I are fused in a spindle shape, but the talus, especially the talus head, of Types I and IV still has a large range of motion, so the degree of flat foot deformity is severer than Type II $(P<0.05)$ (Table 1$)$.

The Gissane's angle, which is formed by the anterior facet and posterior facet of subtalar articular surface, able to reflect the flatness of the STJ and affect talar mobility [31-33]. Type II was significant sharper Type I and Type IV $(P<0.05)$ (Table 2). The result implies that types I and IV have a greater degree of mobility, thereby affecting the surrounding ligamentous structures and this would be expected to make the flat foot deformity severer in Type I and IV $[9,25]$.

Our result showed Type II was sharper Type I and Type IV in the Bohler's angle and is consistent with the severity of the flat foot $\operatorname{deformity}(P<0.05)$ (Table 2 ). Previous studies proved decreased Bohler's angle was closely related to the increased joint pressure which affects the occurrence of osteoarthritis, we therefore believe that the difference in Bohler's angle is more likely to be a secondary change rather than a cause of flat foot deformity.

However, this study still had some limitations. (1) Due to the limitation of the study sample size, severe flat foot was found only in Type I and in none of the other types. (2) We will further analyze the association between the differences in left-right flat foot deformity in the same individual and subtalar articular surface typing in our later study. (3) We measured the morphological parameters by performing CT $3 \mathrm{D}$ reconstruction of the calcaneus, and this method may produce certain errors. 


\section{Conclusion}

After being compared and analyzed the measurement and comparison of morphological parameters, Type I and IV may cause severer flat foot deformity. The area of joint surface area and fusion influenced severity of flat foot deformity by affecting mobility.

\section{Abbreviations}

STJ: Subtalar joint; PTTD: Posterior tibial tendon dysfunction; CT: Computed tomography; 3D: Three-dimensional; SD: Standard deviation.

\section{Acknowledgments}

The authors extend their appreciation to The Affiliated Traditional Chinese Medicine Hospital of Southwest Medical University.

\section{Authors' contributions}

Conceptualization LZ and XP; Methodology XP; Validation SH; Investigation $X Z$; Resources GY.; Writing-Original Draft preparation LZ, XP and XT; Formal analysis BL; Project administration GW; Writing-Reviewing WZ; Editing YY. All authors read and approved the final manuscript.

\section{Funding}

This work was supported by the National Natural Science Foundation of China (Youth Science Foundation Project) (Grant No.82004458), the Traditional Chinese Medicine Research Project of Sichuan Traditional Chinese Medicine Administration (Grant No.2020JC0138), the Health Commission of Sichuan Province Science and Research Project (Popularization and Application Project) (Grant No. 20PJ143), the Luzhou People's GovernmentSouthwest Medical University Shi-zhen Zhong Academician Talent Team Sub-project (Grant No.2018zszysrctdxm), 2020 Orthopedics (Shangantong) special scientific research project of Sichuan Medical Association (Grant No.2020SAT26) and the Southwest Medical University Research Project (Grant No.2020ZRQNA045)

\section{Availability of data and materials}

The datasets used and analysed during the current study are available from the corresponding author on reasonable request.

\section{Declarations}

\section{Ethics approval and consent to participate}

All participants provided verbal informed consent to participate in the study due to our study belonging to retrospective study, we can access the patient's relevant data with the approval of the ethics committee. All procedures were approved by the Ethical Committee of The Affiliated Traditional Chinese Medicine Hospital of Southwest Medical University (No. KY2018043). All methods were performed in accordance with the relevant guidelines and regulations.

\section{Consent for publication}

All study participants provided verbal consent to take part in the study and our study were approved by the Ethical Committee of The Affiliated Traditional Chinese Medicine Hospital of Southwest Medical University (No. KY2018043).

\section{Competing interests}

The authors declare that they have no competing interests.

\section{Author details}

'Department of Orthopedics, The Affiliated Traditional Chinese Medicine Hospital of Southwest Medical University, Luzhou 646000, China. ${ }^{2}$ Center for Orthopedic Diseases Research, The Affiliated Traditional Chinese Medicine Hospital of Southwest Medical University, Luzhou 646000, China. ${ }^{3}$ Expert Workstation in Luzhou, Luzhou 646000, China. ${ }^{4}$ Clinical Base of The Affiliated Traditional Chinese Medicine Hospital of Southwest Medical University, Guangdong Province Medical 3D Printing Application Transformation Engineering Technology Research Center, Luzhou 646000, China. ${ }^{5}$ School of Clinical Medicine, Southwest Medical University, Luzhou 646000, China.

${ }^{6}$ School of Clinical Medicine, Southern Medical University, Guangzhou 510000, China.

Received: 29 July 2021 Accepted: 8 November 2021

Published online: 23 November 2021

\section{References}

1. Tang CYK, Ng KH, Lai J. Adult flatfoot. BMJ. 2020;368:m295.

2. Deland JT. Adult-acquired flatfoot deformity. J Am Acad Orthop Surg. 2008:16:399-406

3. Navarro-Flores E, Losa-Iglesias ME, Becerro-de-Bengoa-Vallejo R, LopezLopez D, Vilar-Fernandez JM, Palomo-Lopez P, et al. Transcultural adaptation and validation of the Spanish Bristol foot score (BFS-S). Aging Dis. 2018;9(5):861-8.

4. Navarro-Flores E, Losa-Iglesias ME, Becerro-de-Bengoa-Vallejo R, ReinaBueno M, Lopez-Lopez D, Romero-Morales C, et al. Cross-cultural adaptation, translation, and validation of the Spanish foot and ankle outcome score questionnaire. Int Wound J. 2020;17(5):1384-90.

5. Krahenbuhl N, Horn-Lang T, Hintermann B, Knupp M. The subtalar joint: a complex mechanism. EFORT Open Rev. 2017;2(7):309-16.

6. Hattori K, Sakuma E, Nakayama M, Kozaki A, Wada I, Otsuka T. An anatomic study of the accessory anterolateral talar facet. Folia Morphol (Warsz). 2015;74(1):61-4.

7. Graham ME, Parikh R, Goel V, Mhatre D, Matyas A. Stabilization of joint forces of the subtalar complex via HyProCure sinus tarsi stent. J Am Podiatr Med Assoc. 2011:101(5):390-9.

8. Gwani AS, Asari MA, Mohd Ismail ZI. How the three arches of the foot intercorrelate. Folia Morphol (Warsz). 2017;76(4):682-8.

9. Kothari A, Bhyva S, Stebbins J, Zavatsky AB, Theologis T. An investigation into the aetiology of flexible flat feet: the role of subtalar joint morphology. Bone Joint J. 2016;98-B(4):564-8.

10. Probasco W, Haleem AM, Yu J, Sangeorzan BJ, Deland JT, Ellis SJ. Assessment of coronal plane subtalar joint alignment in peritalar subluxation via weight-bearing multiplanar imaging. Foot Ankle Int. 2015;36(3):302-9.

11. Ferri M, Scharfenberger AV, Goplen G, Daniels TR, Pearce D. Weightbearing CT scan of severe flexible pes planus deformities. Foot Ankle Int. 2008:29(2):199-204

12. de Cesar NC, Godoy-Santos AL, Saito GH, Lintz F, Siegler S, O'Malley MJ, et al. Subluxation of the middle facet of the subtalar joint as a marker of Peritalar subluxation in adult acquired flatfoot deformity: a case-control study. J Bone Joint Surg Am. 2019;101(20):1838-44.

13. de Cesar NC, Silva T, Li S, Mansur NS, Auch E, Dibbern K, et al. Assessment of posterior and middle facet subluxation of the subtalar joint in progressive flatfoot deformity. Foot Ankle Int. 2020;41(10):1190-7.

14. Yang Y, Cheng HW, Xiong ZR, Liu N, Liu Y, Wang Y, et al. Classification and morphological parameters of the calcaneal Talar facet: which type is more likely to cause osteoarthritis in Chinese population? Biomed Res Int. 2019;2019:6095315.

15. Catanzariti AR, Mendicino RW, Saltrick KR, Orsini RC, Dombek MF, Lamm BM. Subtalar joint arthrodesis. J Am Podiatr Med Assoc. 2005:95(1):34-41.

16. Canavese F, Dimeglio A, Bonnel F. Postoperative CT-scan 3D reconstruction of the calcaneus following lateral calcaneal lengthening osteotomy for flatfoot deformity in children. Is the surgical procedure potentially associated with subtalar joint damage? Foot Ankle Surg. 2018:24(5):453-9.

17. Agarwal S, Garg S, Vasudeva N. Subtalar joint instability and calcaneal spurs associated with the configuration of the articular facets of adult human Calcaneum in Indian population. J Clin Diagn Res. 2016;10(9):AC05-9.

18. Flores DV, Mejia Gomez C, Fernandez Hernando M, Davis MA, Pathria MN. Adult acquired flatfoot deformity: anatomy, biomechanics, staging, and imaging findings. Radiographics. 2019;39(5):1437-60.

19. Smyth NA, Aiyer AA, Kaplan JR, Carmody CA, Kadakia AR. Adult-acquired flatfoot deformity. Eur J Orthop Surg Traumatol. 2017;27(4):433-9.

20. Abousayed MM, Alley MC, Shakked R, Rosenbaum AJ. Adult-acquired flatfoot deformity: etiology, diagnosis, and management. JBJS Rev. 2017:5(8):e7. 
21. Shin HS, Lee JH, Kim EJ, Kyung MG, Yoo HJ, Lee DY. Flatfoot deformity affected the kinematics of the foot and ankle in proportion to the severity of deformity. Gait Posture. 2019;72:123-8.

22. Resnick RB, Jahss MH, Choueka J, Kummer F, Hersch JC, Okereke E. Deltoid ligament forces after tibialis posterior tendon rupture: effects of triple arthrodesis and calcaneal displacement osteotomies. Foot Ankle Int. 1995;16(1):14-20.

23. Bluman EM, Myerson MS. Stage IV posterior tibial tendon rupture. Foot Ankle Clin. 2007;12(2):341-62 viii.

24. Jimenez-Cebrian AM, Becerro-de-Bengoa-Vallejo R, Losa-Iglesias ME, de Labra C, Calvo-Lobo C, Palomo-Lopez P, et al. Kinesiophobia levels in patients with Parkinson's disease: a case-control investigation. Int J Environ Res Public Health. 2021;18(9):4791.

25. Barbaix E, Van Roy P, Clarys JP. Variations of anatomical elements contributing to subtalar joint stability: intrinsic risk factors for post-traumatic lateral instability of the ankle? Ergonomics. 2000;43(10):1718-25.

26. Bruckner J. Variations in the human subtalar joint. J Orthop Sports Phys Ther. 1987:8:489-94

27. Ling SK, Lui TH. Posterior Tibial tendon dysfunction: an overview. Open Orthop J. 2017;11:714-23.
28. Mittlmeier T, Rammelt S. Update on subtalar joint instability. Foot Ankle Clin. 2018;23(3):397-413.

29. Jung MH, Choi BY, Lee JY, Han CS, Lee JS, Yang YC, et al. Types of subtalar joint facets. Surg Radiol Anat. 2015;37(6):629-38.

30. Prasad SA, Rajasekhar SSSN. Morphometric analysis of talus and calcaneus. Surg Radiol Anat. 2019;41(1):9-24.

31. Badalahu QB, Luo J, Zeng Y, Fu S, Zhang L. Classification of the subtalar articular surface and its matching situation: an anatomical study on Chinese subtalar joint. Surg Radiol Anat. 2020;42(10):1133-9.

32. Ragab AA, Stewart SL, Cooperman DR. Implications of subtalar joint anatomic variation in calcaneal lengthening osteotomy. J Pediatr Orthop. 2003;23(1):79-83.

33. Qiang M, Chen Y, Zhang K, Li H, Dai H. Measurement of three-dimensional morphological characteristics of the calcaneus using CT image post-processing. J Foot Ankle Res. 2014;7:19.

\section{Publisher's Note}

Springer Nature remains neutral with regard to jurisdictional claims in published maps and institutional affiliations.
Ready to submit your research? Choose BMC and benefit from:

- fast, convenient online submission

- thorough peer review by experienced researchers in your field

- rapid publication on acceptance

- support for research data, including large and complex data types

- gold Open Access which fosters wider collaboration and increased citations

- maximum visibility for your research: over 100M website views per year

At BMC, research is always in progress.

Learn more biomedcentral.com/submissions 